\title{
Impairment of brain and muscle energy metabolism detected by magnetic resonance spectroscopy in hereditary spastic paraparesis type 28 patients with DDHD1 mutations
}

\author{
Rocco Liguori · Maria Pia Giannoccaro • \\ Alessia Arnoldi - Andrea Citterio - Caterina Tonon • \\ Raffaele Lodi · Nereo Bresolin • Maria Teresa Bassi
}

\begin{abstract}
Mutations in DDHD1 gene have been associated with the SPG28 subtype of Hereditary Spastic Paraparesis (HSP). Clinical phenotype includes axonal neuropathy, distal sensory loss, and cerebellar eye movement disturbances. We screened 96 index subjects from recessive HSP families for mutation and identified one family with two sibs carrying mutations in DDHD1 gene. Clinical, neuropsychological, and neuroimaging studies were performed, including MR spectroscopy of brain and muscle of the two mutated patients. Two novel heterozygous mutations in DDHD1 were found in the affected members of one family, with clinical features overlapping the SPG28 subtype. Of note, MR spectroscopy of brain and
\end{abstract}

R. Liguori ( $\&$ )

IRCCS Istituto delle Scienze Neurologiche di Bologna, Pad G1, Ospedale Bellaria, Via Altura 3, 40139 Bologna, Italy

e-mail: rocco.liguori@unibo.it

R. Liguori . M. P. Giannoccaro _ C. Tonon . R. Lodi Department of Biomedical and NeuroMotor Sciences (DiBiNeM), University of Bologna, Bologna, Italy

A. Arnoldi . A. Citterio . C. Tonon . R. Lodi

Functional MR Unit, S.Orsola-Malpighi Hospital, Bologna, Italy

N. Bresolin . M. T. Bassi

Laboratory of Molecular Biology, Scientific Institute IRCCS

Eugenio Medea, Bosisio Parini (Lecco), Italy

N. Bresolin

Department of Physiopathology and Transplantation, Dino Ferrari Centre, Neurology Unit, IRCCS Ca' Granda, Ospedale Maggiore Policlinico Foundation, University of Milan, Milan, Italy muscle in these patients indicated a mild deficit of brain energy metabolism in the oldest and most severely affected patient, while an impairment of energy metabolism was found in the skeletal muscle of both patients. Unlike the DDHD2 mutated patients, no evidence of lipid accumulation in the brain was found. Our data along with those previously reported suggest a dysfunction in the OXPHOS system possibly due to mitochondrial lipid content modification, which could be a central mechanism in the pathogenesis of SPG28.

Keywords Hereditary spastic paraplegia . SPG28 . Proton magnetic resonance spectroscopy. Phosphorus magnetic resonance spectroscopy

Introduction

Hereditary spastic paraplegia (HSP) is a clinical and genetic heterogeneous group of disorders sharing the common feature of progressive spasticity in the lower extremities. Pure forms are characterized by isolated spinal involvement, while complicated ones show the presence of other neurologic or systemic abnormalities [1]. Among the 71 different subtypes of HSP so far identified [1, 2], a subset of them is associated with defects in genes involved in different aspects of lipid metabolism, such as SPG54, SPG28, SPG56, SPG46, SPG26, and SPG5 [1]. SPG28 was first associated with a pure form of HSP [3], then mutations in the causative gene DDHD1 were found in additional three families [4] presenting with a clinical phenotype, including axonal neuropathy, distal sensory loss, and cerebellar eye movement disturbances [3, 4]. DDHD1 encodes a cytosolic phosphatidic acid-preferring phospholipase A1 (PA-PLA1). Mutations in DDHD1 were demonstrated to 
induce alterations of mitochondrial bioenergetics with increased oxidative stress in patients' lymphoblasts [4]. In a screening for mutations in DDHD1 carried out in 96 index patients from HSP families, we identified one family carrying two novel mutations in this gene. Here we report the detailed clinical and neuropsychological characterization of this family. We also investigated the presence of lipid accumulation and checked the energy metabolism in this form of HSP by brain and muscle magnetic resonance spectroscopy (MRS).

Patients and methods

A series of 96 index patients from families with AR-HSP were clinically evaluated and genetically screened for DDHD1 mutations. Diagnosis was based on Harding's Criteria [5]. Detailed methods are provided in online electronic supplementary files. Screening for DDHD1 (SPG28) mutations led to the identification of one single family carrying mutations in DDHD1, clinically described below.

Genetic analysis

We obtained blood samples and clinical data from affected and unaffected subjects of the pedigree under a protocol of informed consent approved by the Ethics Committee of the E. Medea Scientific Institute. Mutation analysis was performed by targeted resequencing of the coding exons of DDHD1 gene, using an amplicon-based high-throughput pooled-sequencing method for amplicon generation. Technical details are available in online electronic supplementary files. Sanger sequencing was also used to validate all variants identified.

\section{Results}

Mutation analysis

Mutation screening of DDHD1 coding exons in 96 index HSP patients led to the identification of two compound heterozygous mutations in DDHD1 in the two affected sibs of a single family (Fig. 1). A single nucleotide insertion in exon 6 (c.1422_1423insA, p.Val476Serfs*20) and a single nucleotide deletion in exon 11 (c.2279delT- p.Met760Serfs*37) were detected, both leading to early protein truncation either upstream or within the DDHD domain, respectively. Thus, the truncated DDHD1 protein deriving from any of the two alleles is lacking an integral DDHD domain, which is responsible for the phospholipase activity of DDHD1 protein, thereby leading to a likely loss of protein function.
Clinical data of the family carrying the DDHD1 mutation

The family we describe includes two affected subjects ( $\mathrm{pt}$ II-2 and II-3) of three siblings. The parents were unaffected and not consanguineous. Patient II-2 (index) complained progressive gait disturbances since age 15 years due to leg weakness and "stiffness" while walking, and unsteadiness. After 20 years from symptoms onset, he was still able to walk unassisted. Neurologic examination showed mild distal muscle weakness in legs, bilateral lower limb spasticity with brisk tendon reflexes and Babinski sign. Claw foot and lumbar scoliosis were present. Gait was spastic.

Patient II-3 had slowly progressing walking difficulties from age of 16 years. Neurologic examination revealed tongue fasciculations associated with slight wasting and weakness, increased muscle tone, hyperreflexia in the lower limbs with bilateral Babinski sign and spastic gait.

Neurophysiological studies were performed. Concentric needle EMG in upper and lower limb muscles disclosed chronic neurogenic changes in both patients. Electroneurographic studies of the tibial nerves showed slight slowing of motor conduction velocity $(23 \%)$ and marked reduction of compound motor action potential (93\%) in patient II-2; normal findings were detected in patient II-3. Transcranial magnetic stimulation showed abnormal conduction in corticospinal pathways (Table 1). Visual evoked potentials were normal. Sensory evoked potentials (SEPs) were normal in patient II-3, whereas in patient II-2 SEPs to stimulation of posterior tibial nerves at the ankle showed no peripheral responses, absent spinal responses at D10 and increased P40 latency (?12\%) with decreased amplitude of $0.4 \mathrm{IV}$ (normal value $2 \pm 0.5$; mean $\pm 2 \mathrm{SD}$ ).

Neuropsychological studies

Mini mental state evaluation and brief mental deterioration battery scores were normal. However, neuropsychological examination showed deficits in simple analogy tests in both patients and also in phonemic and semantic verbal fluency in patient II-2 (Table 1).

Magnetic resonance imaging and magnetic resonance spectroscopy investigations

Structural brain MRI in both patients did not show any morphology or signal intensity changes. Also, the morphometric and volumetric analysis of cortical and subcortical regions was normal.

Brain ${ }^{1} \mathrm{H}$-MRS in the cerebrospinal fluid of the lateral ventricles detected a mild pathological accumulation of lactate in patient II-2 (Fig. 1), whereas no lactate was found in patient II-3. 


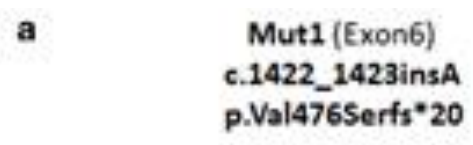

$\overline{A C A A A R K W M S A}$ G T A C 6 A

- Rnanduaras. A C A A A T T A G A

C

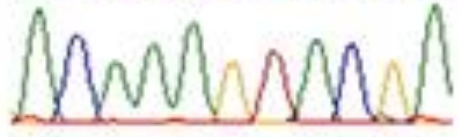
$A \subset A A \& A$ A $A \subset G$

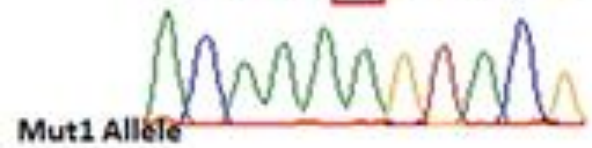

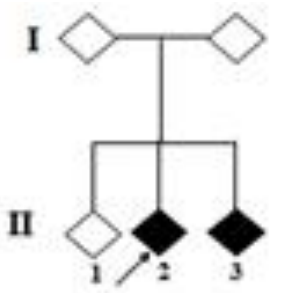

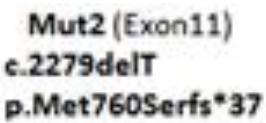

p.Met760Serfs*37

$\overline{A G G A A K K T K K}$ T $\&$ T T
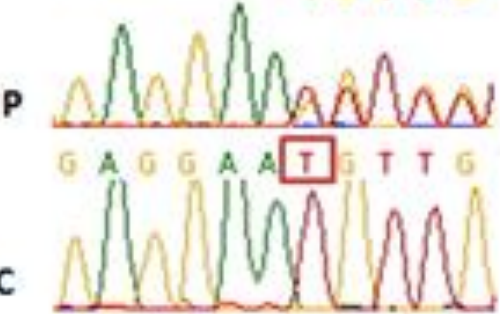

G A G G A A G T T G T

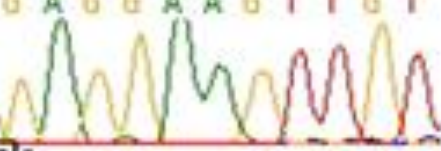

Mut2 Allele

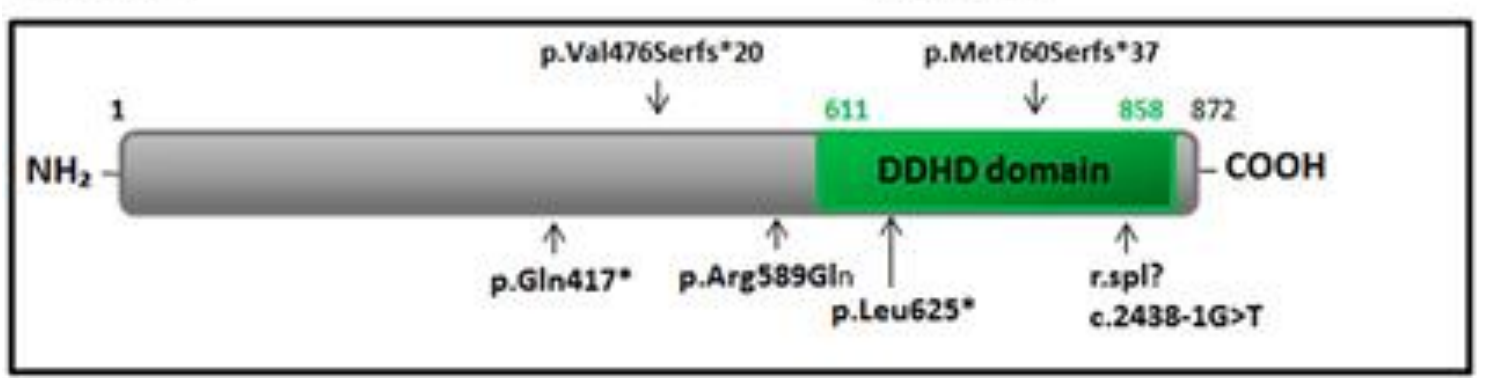

b

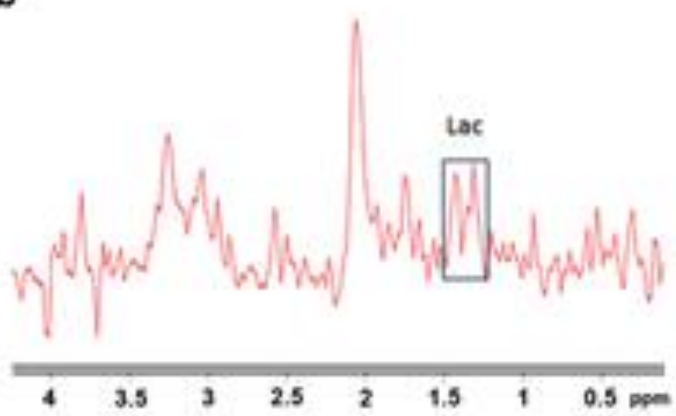

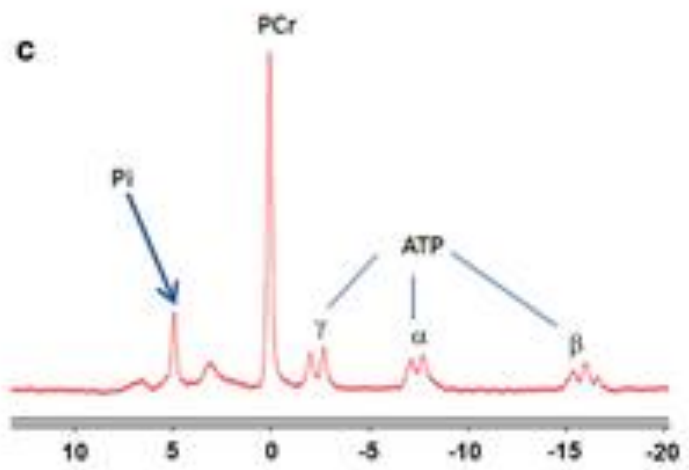

COOH-terminus. In the upper part: the mutations identified in this study; the known mutations are indicated below. $b{ }^{1} \mathrm{H}-\mathrm{MRS}$ spectrum obtained from the CSF of the lateral ventriculi of patient II-2 showing a pathological mild accumulation of lactate (Lac) (rectangle). $c{ }^{31} \mathrm{P}$ MRS spectrum obtained at rest from the left calf muscle of patient II2 showing an increase of Pi peak (arrow). Pi inorganic phosphate, $\mathrm{PCr}$ phosphocreatine, ATP adenosine-triphosphate

Compared to healthy controls, ${ }^{31} \mathrm{P}-\mathrm{MRS}$ of muscle at rest showed a reduction of the phosphocreatine/inorganic phosphate $(\mathrm{PCr} / \mathrm{Pi})$ ratio both in patient II-2 (=3.90 vs. $7.29 \pm 0.89$, mean \pm standard deviation, in healthy subjects) (Fig. 1) and in patient II-3 (=2.80). Muscle pH was normal. 
We describe the clinical, neuroradiological, and neuropsychological features of two affected sibs of an HSP family carrying compound heterozygous frameshift mutations in the DDHD1 gene leading to the loss of the DDHD domain. DDHD1 is a phosphatidic acid-preferring phospholipase A1 and this activity is strictly related to the DDHD domain integrity. Therefore, the mutations here identified should lead to loss of protein function. This family was identified in a mutation screening of DDHD1 carried out in 96 index patients from autosomal recessive HSP families, thus indicating that SPG28 could not be a frequent subtype of either pure or mildly complicated HSP (cHSP).

The clinical history of the DDHD1 mutated patients described here overlaps the one of the previously reported SPG28-related families [3, 4]. It is characterized by an apparently pure phenotype with evidence of pyramidal tract involvement in the second decade of life (median age 15 years) and progressive loss of walking independence. Neurophysiological investigations revealed signs of corticospinal tracts involvement. Neuropsychological testing disclosed subtle deficits in verbal abstract thinking and verbal fluency suggestive of frontal lobe dysfunction [6, 7]. These findings suggest that, also in an apparently pure phenotype, a more widespread pathological involvement could be demonstrated and is concordant with previous studies showing an involvement of the frontal lobe in cHSP [8].

We also assessed the energy metabolism through brain ${ }^{1} \mathrm{H}$ - and muscle ${ }^{31} \mathrm{P}$ - MRS, also looking for the presence of brain accumulation of lipids on the basis of a previous report of an abnormal lipid peak in the brain of patients carrying truncating mutations in the DDHD domain of DDHD2 [4] DDHD2 together with DDHD1 and SEC23IP are members of the mammalian family of intracellular phospholipase A1, sharing a lipase motif $(\mathrm{GxSxG})$ and the DDHD domain [9].

While structural brain MRI in our DDHD1 mutated patients was normal, a mild deficit of brain energy metabolism in the oldest and most severely affected patient II-2 was found, as shown by a pathological accumulation of ventricular lactate. The pathological presence of lactic acid in the cerebrospinal fluid is typically observed in patients with primary mitochondrial diseases [10] and it is secondary to oxidative phosphorylation impairment. In skeletal muscle at rest, in both patients, ${ }^{31} \mathrm{P}$-MRS showed a significant reduction of $\mathrm{PCr}$ to $\mathrm{Pi}$ ratio. Skeletal muscle $\mathrm{PCr} / \mathrm{Pi}$ ratio indicates a reduction of cellular energy reserve (i.e., phosphorylation potential) and is a typical finding in patients with primary mitochondrial myopathies. In contrast with patients carrying mutations in the DDHD2 gene, 
in none of the DDHD1 patients brain lipid accumulation was detected using ${ }^{1} \mathrm{H}-\mathrm{MRS}$.

Although belonging to the same protein family with similar hydrolyzing activity (an acyl group of phospholipids at the sn-1 position) and similarly implicated in organelle biogenesis and membrane trafficking, DDHD1 and DDHD2 show different subcellular localization. DDHD2 is more stably associated with the Golgi/endoplasmic reticulum [11] while DDHD1 is mainly localized in cytosol with a partial localization in microsomes and mitochondria [12]. In addition, DDHD1 has been recently involved in the formation of lysophosphatidylinositol (LPI), and in particular of 2-arachidonoyl LPI [12], a lipid mediator with various physiological functions, including $\mathrm{Ca}^{2}$ mobilization and cell migration and proliferation [13].

This partly explains such different functional consequences deriving from mutations in the DDHD domain of the two proteins, including the different clinical phenotypes (severe and complicated in DDHD2/SPG54 vs. pure or mildly complicated in DDHD1/SPG28 subtype).

The findings here reported, the partial localization of DDHD1 at mitochondrial level, along with the previous demonstration of a reduction of mean mitochondrial respiration activities and ATP levels, together with an increment of cytosolic hydrogen peroxide in SPG28 lymphoblasts compared to controls [4] overall suggest a dysfunction in the OXPHOS system, possibly due to mitochondrial lipid content modification. Mitochondrial phospholipids, including phosphatidic acid, are involved in different processes such as mitochondrial membrane integrity and morphology maintenance, fusion and fission, protein import, and apoptosis [14]. Mitochondrial dynamics, through fusion and fission, is fundamental for mitochondrial function and may be affected by alteration of lipid content [15].

The mitochondrial dysfunctions occurring in both brain and skeletal muscle of DDHD1 patients here reported could likely represent a central mechanism in the pathogenesis of SPG28.

Acknowledgments The research was supported by funds from the Italian Ministry of Health (RC2013-2014 to MTB). We thank the patients and their families for the cooperation and participation in the study. We thank Miss C. Baroncini for the English editing.

Conflicts of interest The authors declare that they have no conflict of interest.

Ethical standard The study was approved by the Ethics Committee of the E. Medea Scientific Institute and has therefore been performed in accordance with the ethical standards laid down in the 1964
Declaration of Helsinki and its later amendments.All patients consented for their data to be used for research.

\section{References}

1. Fink JK (2013) Hereditary spastic paraplegia: clinico-pathologic features and emerging molecular mechanisms. Acta Neuropathol 126:307-328

2. Novarino G, Fenstermaker AG, Zaki MS et al (2014) Exome sequencing links corticospinal motor neuron disease to common neurodegenerative disorders. Science 343:506-511

3. Bouslam N, Benomar A, Azzedine H et al (2005) Mapping of a new form of pure autosomal recessive spastic paraplegia (SPG28). Ann Neurol 57:567-571

4. Tesson C, Nawara M, Salih MA et al (2012) Alteration of fattyacid-metabolizing enzymes affects mitochondrial form and function in hereditary spastic paraplegia. Am J Hum Genet 91:1051-1064

5. Harding AE (1983) Classification of the hereditary ataxias and paraplegias. Lancet 1:1151-1155

6. Costafreda SG, Fu CH, Lee L et al (2006) A systematic review and quantitative appraisal of fMRI studies of verbal fluency: role of the left inferior frontal gyrus. Hum Brain Mapp 27:799-810

7. Green AE, Fugelsang JA, Kraemer DJ et al (2006) Frontopolar cortex mediates abstract integration in analogy. Brain Res 1096:125-137

8. Uttner I, Baumgartner A, Sperfeld AD, Kassubek J (2007) Cognitive performance in pure and complicated hereditary spastic paraparesis: a neuropsychological and neuroimaging study. Neurosci Lett 419:158-161

9. Schuurs-Hoeijmakers JH, Geraghty MT, Kamsteeg EJ et al (2012) Mutations in DDHD2, encoding an intracellular phospholipase $\mathrm{A}(1)$, cause a recessive form of complex hereditary spastic paraplegia. Am J Hum Genet 91:1073-1081

10. La Morgia C, Achilli A, Iommarini L et al (2008) Rare mtDNA variants in Leber hereditary optic neuropathy families with recurrence of myoclonus. Neurology 70:762-770

11. Inoue H, Baba T, Sato S et al (2012) Roles of SAM and DDHD domains in mammalian intracellular phospholipase A1 KIAA0725p. Biochim Biophys Acta 1823:930-939

12. Yamashita A, Kumazawa T, Koga H et al (2010) Generation of lysophosphatidylinositol by DDHD domain containing 1 (DDHD1): possible involvement of phospholipase D/phosphatidic acid in the activation of DDHD1. Biochim Biophys Acta 1801:711-720

13. Yamashita A, Oka S, Tanikawa T et al (2013) The actions and metabolism of lysophosphatidylinositol, an endogenous agonis1 for GPR55. Prostaglandins Other Lipid Mediat 107:103-116

14. Osman C, Voelker DR, Langer T (2011) Making heads or tails of phospholipids in mitochondria. J Cell Biol 192:7-16

15. Steenbergen R, Nanowski TS, Beigneux A et al (2005) Disruption of the phosphatidylserine decarboxylase gene in mice causes embryonic lethality and mitochondrial defects. J Biol Chem 280:40032-40040

16. Gallassi R, Bisulli A, Oppi F et al (2008) Subjective cognitive complaints, neuropsychological performance, affective and behavioural symptoms in non-demented patients. Int $\mathbf{J}$ Geriatı Psychiatry 23:95-101 